Internist 2010 $\cdot 51: 813-814$

DOI 10.1007/s00108-010-2633-2

Online publiziert: 12. Mai 2010

(c) Springer-Verlag 2010

\author{
B.E. Strauer \\ Medizinische Klinik und Poliklinik, Heinrich-Heine-Universität Düsseldorf
}

\title{
Das Herz bei internistischen Erkrankungen
}

lung einer Herzerkrankung auf die Möglichkeit der Behandlung der internistischen Grundkrankheit zu konzentrieren. Viele Patienten mit sog. therapierefraktären Herzerkrankungen könnten wir erfolgreicher behandeln, wenn die Ursachenfindung im Sinne der extrakardialen Krankheitsidentifizierung mit entsprechender nachfolgender Kausaltherapie wirksam umgesetzt würde. Dies ist ein immer wieder zu wenig beachtetes Feld in der Diagnostik und Therapie kardialer Erkrankungen, die oft nur mit symptomatischen Maßnahmen angegangen werden.

Das vorliegende Heft „Das Herz bei internistischen Erkrankungen" stellt die Fortsetzung der bereits 2007 begonnenen Thematik dar, mit dem Ziel, die Auswirkungen allgemein-internistischer Erkrankungen auf das Herz, insbesondere auf seine Funktion, die Diagnostik sowie die spezifischen Therapiemöglichkeiten darzustellen. In 6 Beiträgen werden wichtige Themen zur Syntropie zwischen allgemein-internistischen sowie kardialen Erkrankungen fokussiert, von denen sich einige der vielen dargebotenen relevanten lassen:

Etwa 20\% der Bevölkerung westlicher Industrienationen haben einen Bluthochdruck: $70 \%$ aller 70-Jährigen sind hochdruckkrank. Zum Zeitpunkt der Hochdruckdiagnose weisen etwa die Hälfte bis zwei Drittel der Hochdruckpatienten eine hypertensiv bedingte Herzhypertrophie bzw. Herzinsuffizienz auf. Der Bluthochdruck ist als Volkskrankheit der Prototyp einer primär extrakardialen Erkrankung mit bevorzugt kardialer Beteiligung und ein gravierender Risikofaktor für Herzerkrankungen (Herzhypertrophie, Herzin- suffizienz, koronare Herzkrankheit; s. Beitrag Hennersdorf, Motz, Schannwell).

Patienten mit koronarer Herzkrankheit zeigen oft psychosomatische Begleiterkrankungen wie auch Patienten mit psychischen Erkrankungen vermehrt zu kardialen Krankheitsmanifestationen neigen. Soziale Isolation, inadäquater sozioökonomischer Status, Gratifikationskrisen [2], depressive Fehlverarbeitungen etc. spielen eine Rolle. Die früher vermehrte Inzidenz des Herzinfarkts bei „Managern“ ist einer deutlichen Beteiligung von Patienten mit niedrigem sozioökonomischem Status gewichen. In allen Fällen kommt es besonders auf die psychosomatische Gesamtdiagnose und Therapie an (s. Beitrag Herrmann-Lingen und Meinertz).

Rund $10-20 \%$ aller Patienten mit virologisch-bioptisch gesicherter Myokarditis entwickeln eine meist mit zeitlicher Latenz einhergehende Herzinsuffizienz bzw. chronische dilatative Herzmuskelerkrankung. Ein ursächlicher Zusammenhang mit der Fülle der möglichen Virusinfektionen durch systemische wie auch kardiotrope Erreger gilt als gesichert: Entero-, Adeno- und Zytomegalieviren, Parvovirus B19, Epstein-Barr-Virus, HHV6 wie auch $\mathrm{H}_{1} \mathrm{~N}_{1}$ kommen u. a. in Betracht. Dabei kann die virale Manifestation kardial spezifisch sein, aber auch als Zweiterkrankung auftreten. Genaue Diagnose- und Behandlungspfade sind hier entscheidend (s. Beitrag Pankuweit und Maisch).

Die Sepsis ist eine systemische Entzündungsreaktion als Antwort auf einen infektiösen Stimulus mit den Kriterien des SIRS, des „systemic inflammatory response syndrome “. Bei fortgeschrittener Sepsis liegt regelhaft eine Herzbeteiligung vor. Als prognoserelevanter Parameter kommt es zu einer Einschränkung funktion zu erreichen. Dennoch ist es besonders wichtig, sich bei jeder Behand- 
der Herzfrequenzvariabilität. Dieses Phänomen entspricht der bei Sepsis ausgeprägten autonomen Dysfunktion, die mit dem Schweregrad der Sepsis, d. h. dem Ausmaß des septischen Multiorgandysfunktionssyndroms korreliert (s. Beitrag Ebelt und Werdan).

Herzpatienten mit Nierenerkrankungen sowie Nierenkranke mit Herzerkrankungen weisen erhöhte Morbiditäten und Mortalitäten auf. Die Interaktionen zwischen Herz und Nieren wie auch zwischen den intendierten therapeutischen Maßnahmen sind vielfältig. Renale Anämie, kardiorenales Syndrom und kardiovaskuläre Risikofaktoren (arterielle Hypertonie, Diabetes mellitus, Dyslipidämien, Zigarettenrauchen) sind als risikoerhöhende Faktoren zu nennen. Die funktionellen und organischen Auswirkungen auf das „kontralaterale“ Organ sind zu berücksichtigen, wie u. a. Verschlechterung der Nierenfunktion unter dem Einfluss von ACE-Hemmern, Wirkungsverlust von Statinen bei Dialysepflicht etc. (s. Beitrag Kiski und Reinecke).

Der für manche medizinische Disziplinen gültige Leitsatz ,in somno securitas“ ist leider für internistisch und kardial erkrankte Patienten nicht immer anzuwenden. Bei atmungsbezogenen Schlafstörungen kommt es zu einer Vielzahl von Störungen des kardiovaskulären Systems wie Hypertonie, Hochdruckkrisen, Hypoxämie, Herzrhythmusstörungen, zerebrovaskulären Durchblutungsstörungen. Andererseits können aber diese Störungen zu kreislaufrelevanten Schlafstörungen führen, sodass oft eine wechselseitige Krankheitsverknüpfung vorliegt. Im therapeutischen Umkehrschluss lassen sich aber auch schlafbezogene Störungen und deren kardiovaskuläre Auswirkungen durch eine z. B. apparativ herbeigeführte Normalisierung der Atmungsfunktion (CPAP, BIPAP) beheben, die somit eine Alternative zur medikamentösen Behandlung von Hochdruck, Herzinsuffizienz und/oder Herzrhythmusstörungen darstellt (s. Beitrag Steiner).

In der vorliegenden Ausgabe von Der Internist ist es naturgemäß nicht möglich, die Vielzahl aller extrakardialen Erkrankungen sowie die Syntropismen zwischen allgemein-internistischen und speziellkardialen Erkrankungen darzustellen. Da- her haben wir die Darstellung auf einige besonders wichtig erscheinende Krankheitsbilder begrenzt, um einen breiten Leserkreis anzusprechen, der alle internistischen Kollegen einschließt.

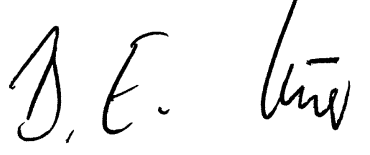

B.E. Strauer

\section{Korrespondenzadresse}

\section{Prof. Dr. B.E. Strauer}

Medizinische Klinik und Poliklinik, Heinrich-Heine-Universität Düsseldorf Moorenstraße 5, 40225 Düsseldorf strauer@med.uni-duesseldorf.de

\section{Literatur}

1. Braunwald E (2007) Heart diseases. Saunders, Philadelphia

2. Siegrist J (1996) Adverse health effects of high-effort/low reward conditions. J Occupat Health Psychol 1: 27-41

3. Strauer BE, Hennersdorf M (2005) 180. Tagung der Rheinisch-Westfälischen Gesellschaft für Innere Medizin. Med Klin (Suppl 1): 1-343
Klinisches Register ",RaFTinG" zur Optimierung der Flüssigkeits- und Volumentherapie auf deutschen Intensivstationen

Unter der Leitung von Prof. Hugo Van Aken (Universitätsklinikum Münster) und Prof. Bernhard Zwißler (Klinikum der Universität München) wird bis zum 28.2.2011 das prospektive klinische Register RaFTinG (Rational Fluid Therapy in Germany, Trial Registration Number NCT01122277) zur Dokumentation der aktuellen infusionstherapeutischen Praxis auf deutschen Intensivstationen durchgeführt. 90 Tage nach Entlassung werden durch ein Followup auch Daten in anonymisierter Form zum Langzeit-Outcome erhoben, welche von den teilnehmenden Kliniken zur internen Qualitätskontrolle genutzt werden können. Die Datenerfassung erfolgt internet-basiert direkt auf Station. Zur Mitwirkung sind alle deutschen Kliniken mit Intensivstation eingeladen.

Weitere Informationen erhalten Sie unter www.rafting-register.de.

Quelle: www.rafting-register.de 